\title{
Childhood snoring: ENT approach and examination
}

\author{
J. Briffod
}

Pediatric ENT physician, Child and Adolescent Specialized Medical Center (CMSEA)

\begin{abstract}
Childhood snoring is caused the narrowness of the upper airway, mainly due to an adenotonsillar hypertrophy. It may impact the quality of life, neurocognitive development and growth. ENT clinical examination is one of the essential components of therapeutic decision-making, taking account of the severity and duration of obstructive signs, presence of complications and, in some cases, sleep recording data, weighed against operative risk. The new technique of radiosurgical partial tonsillectomy has modified this trade-off by considerably decreasing the surgical risk of bleeding and pain.
\end{abstract}

KEY WORDS

Snoring, child, apnea, tonsils

The main problem in case of childhood snoring is to be sure that the symptom can be related to the potential complications before making a decision concerning the treatment, and notably the tonsillectomy. However, neither interview, clinical examination nor polysomnography (PSG) allow certainty on this point. Decision is founded on a range of input, of which ENT examination is an essential component, locating the airway stenosis.

Snoring is a form of sleep-disordered breathing with consequences ranging from a simple impairment of quality of life (daytime fatigue, nocturnal awakening) to a full obstructive sleep apnea syndrome (OSAS), with its known impact on neurocognitive development (concentration and/or memory disorder), growth and cardiovascular health. O'Brien ${ }^{4}$, in 2004, showed that simple snoring with nocturnal awakening may impair cognitive test results. Some children also present major clinical signs (disturbed sleep, fatigue) but with normal PSG, probably due to upper airway resistance syndrome (UARS), the consequences of which may resemble those of OSAS. Furthermore, the hourly apnea/hypopnea index $(\mathrm{AHI} / \mathrm{h})$ is of limited use, as it is not able to predict clinical complications in a linear manner, although recent studies found that $\mathrm{AHI} / \mathrm{h}$ $>5$ increased cardiovascular risk ${ }^{2}$. The limitation of PSG is that it represents only apnea at a given time $T$, and fails to assess 
progression. Symptom progression is closely related to adenotonsillar growth, which is the main cause of childhood snoring; it is physiological between the ages of 2 and 6 years, related to "adaptation disease", and often regresses after 6 years. These clinical findings were confirmed by the American CHAT study ${ }^{3}$ of a pediatric cohort, which published its first results recently, reporting spontaneous reduction in clinical and PSG sleep apnea symptoms, without treatment, in $46 \%$ of children with mild to moderate OSAS at 7 months' follow-up.

Finally, therapeutic decision-making is founded on a range of clinical evidence including nocturnal signs of obstruction (snoring, apnea, oral breathing, head in hyperextension), sleep impairment, daytime repercussions (daytime fatigue, behavioral disorders), pre-existing complications (neurocognitive disorder, retarded growth), and also symptom progression and the parents' worries. These signs are to be combined with the results of clinical examination, which principally explores for the anatomic location of obstruction. In case of clear signs of obstruction associated with adenotonsillar hypertrophy, PSG is recommended by the French ENT Society ${ }^{4}$ and Health Authority (HAS) only after adenotonsillectomy, given the limitations associated with this examination: availability, cost, technical issues. However, PSG is indicated in case of discordance between interview and clinical findings (no adenotonsillar hypertrophy but clinical signs of obstruction, or severe adenotonsillar hypertrophy without signs of obstruction), associated comorbidity or hemostasis disorder (Fig. 1).

In collating the evidence, clinical ENT examination is of prime importance, and first explores for adenotonsillar hypertrophy, the most frequent cause of childhood snoring. During examination, the tongue depressor should be used with caution, as it may trigger reflex nausea, aggravating the hypertrophy. It should be borne in mind that tonsil size does not systematically correlate with the severity of the clinical signs.

In the absence of adenotonsillar hypertrophy, flexible endoscopy (Fig. 2) should be performed under local anesthesia to study the entire airway from nasal orifice to larynx, exploring the turbinates (especially inferior), nasal septum and choanae. It can detect adenoid hypertrophy (Fig. 3) in the nasopharynx, with greater sensitivity and less radiation than X-ray.

It may also reveal an hypertrophy of the inferior pole of the tonsils overlooked on oral examination ${ }^{5}$. Study of the base of the tongue and larynx is concluded by endoscopy to screen for pharyngeal tumor, tongue-base cyst or laryngomalacia.

Nasal respiration is assessed by checking the displacement of cotton wool or fogging on a metal mirror under the nose after asking the child to breath by the mouth.

In some cases, snoring is one aspect of a malformation syndrome, obesity or Down's syndrome. Flexible endoscopy is indispensable in these cases, to explore for areas of multiple airway narrowing. Pierre Robin sequence associates glossoptosis, retrognathia and cleft palate. The Apert 


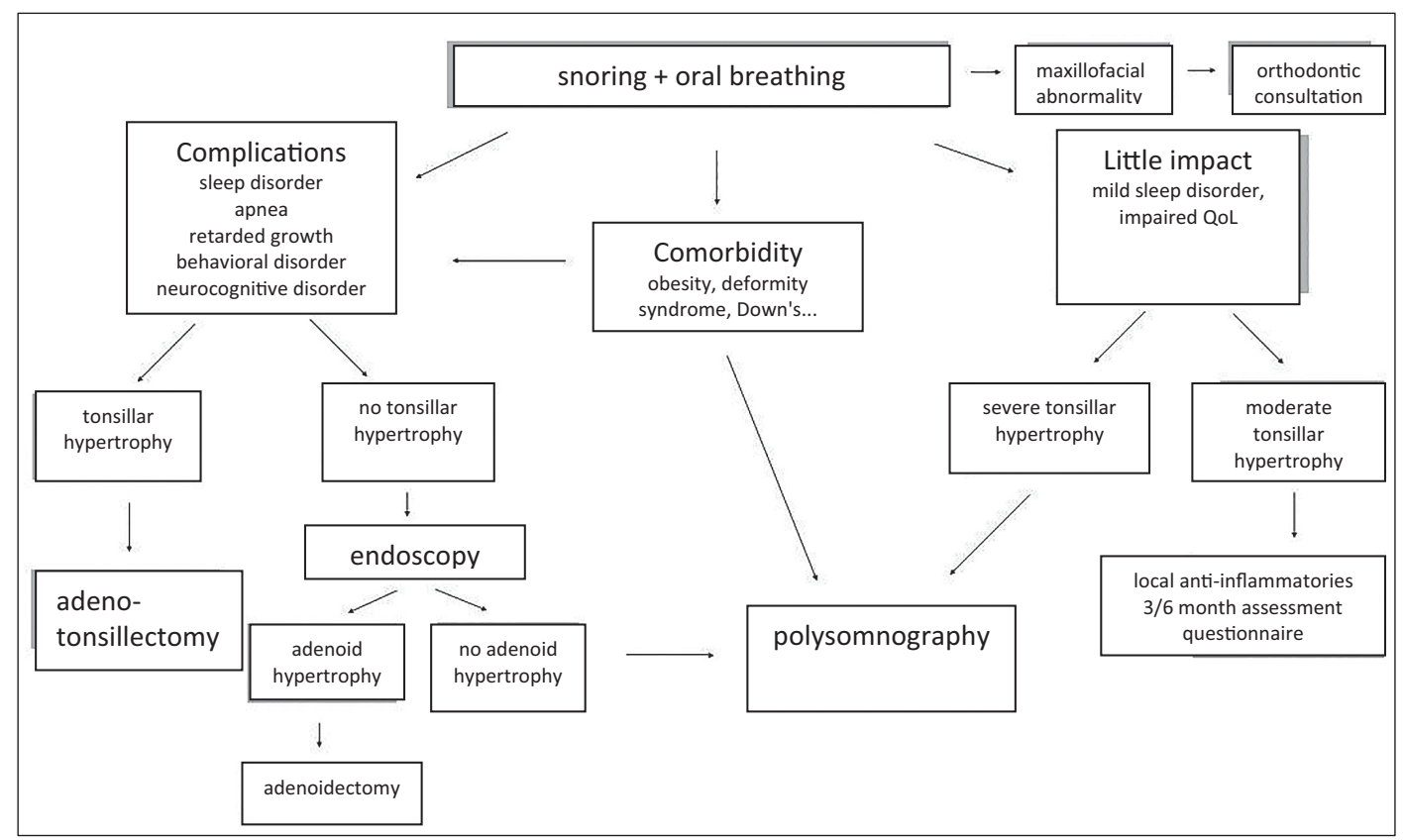

Figure 1

ENT flowchart in childhood snoring.

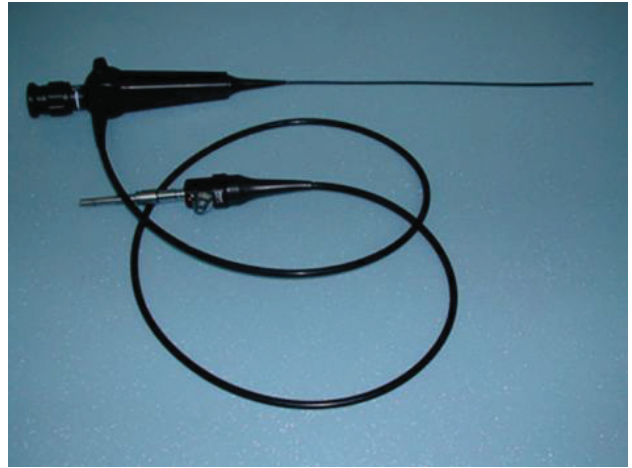

Figure 2

Pediatric flexible endoscope.

and Crouzon syndromes involve abnormalities of the mid-third of the face, especially maxillary hypoplasia. In Down's syndrome, macroglossia should be screened for.

Finally, the ENT physician should be alert to cleft uvula or submucosal

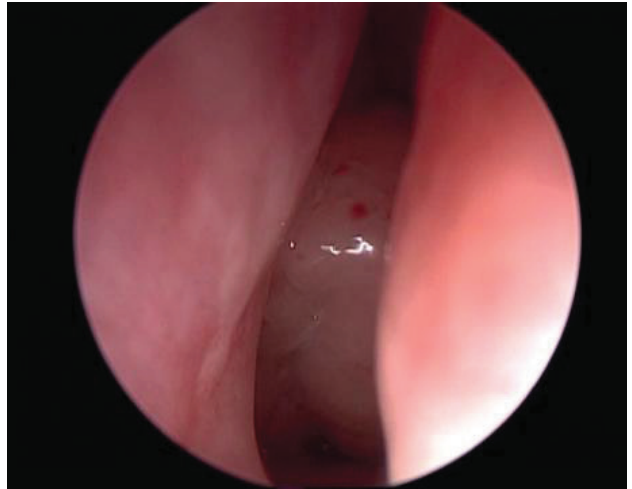

Figure 3

Adenoid hypertrophy.

cleft palate aggravating the risk of velar insufficiency following tonsillar or adenoid surgery.

Paraclinically, CT is reserved to cases of malformation syndrome, absence of adenotonsillar hypertrophy and complex pathology. 
In case of failure of adenotonsillectomy, flexible endoscopy is indispensable, detecting adenoid regrowth or reactive hypertrophy of the lingual tonsils, although the airway obstruction is often not clearly seen.

In conclusion, signs of obstruction and complications associated with tonsillar hypertrophy are often enough to guide decision-making. The critical issue is to make the "right decision" (to operate or not) at the "right time" (to wait or not). The decision takes on board strong social expectations in terms of quality of life and unknowns regarding progression and risk of complications, critically weighing operative risk (anesthesia, pain, bleeding) against expected benefit. New radiosurgical partial tonsillectomy techniques (Fig. 4) have

shifted the criteria of decision-making

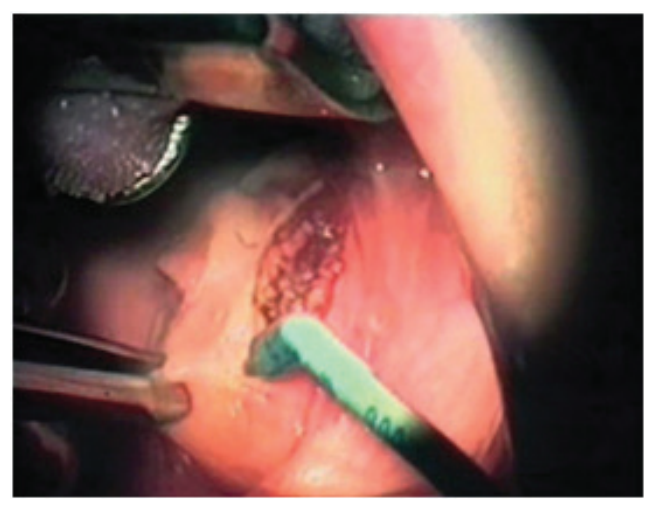

Figure 4

Radiosurgical partial tonsillectomy.

toward considerations of quality of life, as the risk associated with bleeding has been drastically reduced.

Conflict of interest: The author declares no conflicts of interest.

\section{REFERENCES}

1. Couloignier $\mathrm{V}$. Ronflement de l'enfant : un symptôme fréquent à ne pas négliger. Les Entretiens de Bichat 2012;359-64.

2. Lescanne E, et al. Pediatric tonsillectomy: clinical prac- tice guidelines. Eur Ann Otorhinolaryngol Head Neck Dis 2012;129:264-71.

3. Marcus $\mathrm{CL}$, et al. A randomized trial of adenotonsil- lectomy for childhood sleep apnea. N Engl J Med 2013;368:2366-76.

4. O'Brien LM, et al. Neurobehavioral implications of habitual snoring in children. Pediatrics 2004;114:44-9.

5. Vecchierini MF, Monteyrol PJ. Syndrome d'apnées obstructives de l'enfant : nouvelles approches thérapeutiques. Mt Pédiatrie 2013;16:108-17. 